\title{
BIFRACTIONAL BROWNIAN MOTION: EXISTENCE AND BORDER CASES*
}

\author{
Mikhail Lifshits ${ }^{1,2}$ And Ksenia Volkova ${ }^{1}$
}

\begin{abstract}
Bifractional Brownian motion (bfBm) is a centered Gaussian process with covariance

$$
R^{(\mathrm{H}, \mathrm{K})}(s, t)=2^{-\mathrm{K}}\left(\left(|s|^{2 \mathrm{H}}+|t|^{2 \mathrm{H}}\right)^{\mathrm{K}}-|t-s|^{2 \mathrm{HK}}\right), \quad s, t \in \mathbb{R} .
$$

We study the existence of bfBm for a given pair of parameters $(\mathrm{H}, \mathrm{K})$ and encounter some related limiting processes.
\end{abstract}

Mathematics Subject Classification. 60G15, 42A82.

Received February 10, 2015. Revised November 5, 2015.

\section{INTRODUCTION}

Classical fractional Brownian motion $(\mathrm{fBm}) W^{(\mathrm{H})}(t), t \in \mathbb{R}$, with parameter $\mathrm{H} \in(0,1]$, a centered Gaussian process with covariance

$$
R_{W}^{(\mathrm{H})}(s, t):=\frac{1}{2}\left(|s|^{2 \mathrm{H}}+|t|^{2 \mathrm{H}}-|t-s|^{2 \mathrm{H}}\right),
$$

is so widely known and used that it needs no further recommendations. The remarkable properties of this class of processes are described e.g. in ([17], Sect. 7.2) and in ([8], Chap. 4).

Houdré and Villa [9] introduced an extension of fBm called bifractional Brownian motion (bfBm) as a centered Gaussian process $\mathbf{B}^{(\mathrm{H}, \mathrm{K})}$ on $\mathbb{R}$ with covariance

$$
R_{\mathrm{B}}^{(\mathrm{H}, \mathrm{K})}(s, t):=2^{-\mathrm{K}}\left(\left(|s|^{2 \mathrm{H}}+|t|^{2 \mathrm{H}}\right)^{\mathrm{K}}-|t-s|^{2 \mathrm{HK}}\right) .
$$

Usual fBm shows up here when $\mathrm{K}=1$.

There is one more special case of bfBm directly related to the usual fBm. Consider an anti-symmetrized version of $\mathrm{fBm}$,

$$
V^{(\mathrm{H})}(t):=W^{(\mathrm{H})}(t)-W^{(\mathrm{H})}(-t), \quad t \geq 0 .
$$

Keywords and phrases. Bifractional Brownian motion, Gaussian process, fractional Brownian motion.

* The work was supported by Grants NSh.2504.2014.1, RFBR 13-01-00172, and SPbSU 6.38.672.2013.

1 St. Petersburg State University, 28 Stary, Peterhof, Bibliotechnaya pl.,2, 198504 St. Petersburg, Russia.

mikhail@lifshits.org; efrksenia@gmail.com

2 MAI, Linköping University, 58183 Linköping, Sweden. 
It is easy to find its covariance

$$
R_{V}^{(\mathrm{H})}(s, t)=(s+t)^{2 \mathrm{H}}-|t-s|^{2 \mathrm{H}}, \quad s, t \geq 0 .
$$

By comparing this formula with (1.2), we see that $\operatorname{bfBm} \mathbf{B}^{(1 / 2, \mathrm{~K})}, 0<\mathrm{K}<2$, consists, up to a scaling factor, of the two independent versions of $V^{(\mathrm{K} / 2)}$, - one for positive, another for negative times ${ }^{3}$.

Houdré and Villa motivate bfBm just by saying that "usual fBm seems to be a valuable model for small increments (of real processes), but it appears to be inadequate for large increments. It is thus very natural to explore the existence of processes which keep some of the properties of fBm but also enlarge our modelling tool kit". Marouby [15] confirmed this deep guess by showing how a family of bfBm's $\mathrm{H}=\frac{1}{2}, \mathrm{~K} \in(0,1)$, naturally appears as a limit in Mandelbrot micropulse model (see also [11], Sect. 14). On the other hand, in [13] bfBm was used for proving new probabilistic inequalities.

Initially, Houdré and Villa proved the existence of bfBm on $\mathbb{R}$ for

$$
0<\mathrm{H} \leq 1,0<\mathrm{K}<1 .
$$

Later on, Bardina and Es-Sebaiy [2] enlarged the zone of existence. Using an idea of Lei and Nualart [10], they proved that bfBm exists on $\mathbb{R}$ for

$$
0<\mathrm{H} \leq 1,0<\mathrm{K} \leq \min \left\{2, \frac{1}{\mathrm{H}}\right\} .
$$

To the moment when we started this work, it was still unknown whether bfBm exists for any other pairs (H, K). We show below in Proposition 3.1 that conditions $\mathrm{K} \leq 2$ and $\mathrm{HK} \leq 1$ are necessary for the existence of bfBm on $\mathbb{R}_{+}$.

In the zone $\mathrm{H}>1,0<\mathrm{K}<\frac{1}{\mathrm{H}}$, which is most difficult for the research, we proceed with spectral analysis and trace a new numerical bound between the zones of existence and non-existence. We are guided by a guess of D.S. Egorov who conjectured that for any fixed $\mathrm{H} \geq 1$ there exists a positive $\overline{\mathrm{K}}(\mathrm{H})<\mathrm{H}^{-1}$ such that bfBm exists for $(\mathrm{H}, \mathrm{K})$ with any $\mathrm{K}<\overline{\mathrm{K}}(\mathrm{H})$ and does not exist for any $\mathrm{K}>\overline{\mathrm{K}}(\mathrm{H})$.

\section{Existence ARGUMENTS}

For reader's convenience, we briefly recall here (and extend) the key arguments from [9] for the case $0<\mathrm{K}<1$, and those from $[2,10,14]$ for the case $1<\mathrm{K} \leq 2$, proving the existence of bfBm.

\subsection{Case $0<\mathrm{K}<1$}

The arguments of Houdré and Villa actually have nothing to do with fBm or bfBm, as the following statement shows.

Recall that a Bernstein function is a function $f: \mathbb{R}_{+} \rightarrow \mathbb{R}_{+}$which admits the following Lévy-Khintchine representation

$$
f(\lambda)=a+b \lambda+\int_{0}^{\infty}\left(1-\mathrm{e}^{-x \lambda}\right) \mu(\mathrm{d} x)
$$

where $a, b \geq 0$ are some constants and $\mu$ is a measure on $(0, \infty)$ satisfying the integrability condition

$$
\int \min \{x, 1\} \mu(\mathrm{d} x)<\infty .
$$

Bernstein functions, many examples and their connections to various fields of mathematics are discussed in the monograph [16]. Typical examples are $\lambda \rightarrow \log (1+\lambda)$ and $\lambda \rightarrow \lambda^{\mathrm{K}}$ for $0<\mathrm{K} \leq 1$. If $0<\mathrm{K}<1$, the representation (2.1) takes the form

$$
\lambda^{\mathrm{K}}=\frac{\mathrm{K}}{\Gamma(1-\mathrm{K})} \int_{0}^{\infty}\left(1-\mathrm{e}^{-x \lambda}\right) x^{-1-\mathrm{K}} \mathrm{d} x .
$$

\footnotetext{
${ }^{3}$ We did not find this identification with anti-symmetrized $\mathrm{fBm}$ in the literature, although the sister object, a symmetrized fBm $W^{(\mathrm{H})}(t)+W^{(\mathrm{H})}(-t)$, appears in [4] under the name of sub-fractional Brownian motion in connection to the limiting behavior of occupation time of particle systems.
} 
Proposition 2.1. Let $Y(t), t \in \mathbb{R}$ be a centered process with stationary increments and finite second moments

$$
\sigma(t)^{2}:=\mathbb{E} Y(t)^{2}
$$

Then for any Bernstein function $f(\cdot)$ there exists a process with covariance

$$
R_{f, \sigma}(s, t):=f\left(\sigma(s)^{2}+\sigma(t)^{2}\right)-f\left(\sigma(s-t)^{2}\right), \quad s, t \in \mathbb{R} .
$$

Remark 2.2. For $\mathrm{fBm} Y=W^{(\mathrm{H})}$ we have $\sigma(t)=|t|^{\mathrm{H}}$, thus Proposition 2.1 used with $f(\lambda)=\lambda^{\mathrm{K}}$ proves the existence of bfBm with $0<\mathrm{H}, \mathrm{K} \leq 1$.

Proof. For $f(\lambda)=a+b \lambda$ we simply have

$$
\begin{aligned}
R_{f, \sigma}(s, t) & =b\left[\sigma(s)^{2}+\sigma(t)^{2}-\sigma(s-t)^{2}\right] \\
& =b\left[\mathbb{E} Y(s)^{2}+\mathbb{E} Y(t)^{2}-\mathbb{E}(Y(t)-Y(s))^{2}\right] \\
& =2 b \mathbb{E} Y(s) Y(t):=2 b R_{Y}(s, t) .
\end{aligned}
$$

Therefore, the process $\tilde{Y}(t):=\sqrt{2 b} Y(t)$ solves the problem.

Let now $a=b=0$. In view of the formula (2.1), it is sufficient to find a process on $\mathbb{R}$ with covariance

$$
\begin{aligned}
R_{x}^{(\sigma)}(s, t) & :=\left(1-\exp \left(-x\left(\sigma(s)^{2}+\sigma(t)^{2}\right)\right)-\left(1-\exp \left(-x \sigma(t-s)^{2}\right)\right)\right. \\
& =\exp \left(-x \sigma(t-s)^{2}\right)-\exp \left(-x\left(\sigma(s)^{2}+\sigma(t)^{2}\right)\right) \\
& =\exp \left(-x \sigma(s)^{2}\right) \exp \left(-x \sigma(t)^{2}\right)\left[\exp \left(x\left(\sigma(s)^{2}+\sigma(t)^{2}-\sigma(t-s)^{2}\right)\right)-1\right] \\
& =\exp \left(-x \sigma(s)^{2}\right) \exp \left(-x \sigma(t)^{2}\right)\left[\exp \left(2 x R_{Y}(s, t)\right)-1\right] \\
& =\exp \left(-x \sigma(s)^{2}\right) \exp \left(-x \sigma(t)^{2}\right) \sum_{m=1}^{\infty} \frac{(2 x)^{m}}{m !} R_{Y}(s, t)^{m}
\end{aligned}
$$

for any $x>0$. The latter clearly exist along with processes having covariances $R_{Y}(\cdot, \cdot)^{m}$.

\subsection{Case $1<\mathrm{K} \leq 2$}

Following Lei and Nualart [10], consider the real Gaussian process

$$
X_{0}^{(\mathrm{K})}(t):=\int_{0}^{\infty}\left(1-\mathrm{e}^{-r t}\right) r^{-(1+\mathrm{K}) / 2} \mathcal{W}(\mathrm{d} r), \quad t \geq 0,
$$

where $\mathcal{W}$ is an appropriate uncorrelated Gaussian noise. The process $X^{(\mathrm{K})}$ is well defined for $\mathrm{K} \in(0,2)$. By using (2.2), and analogous formula for $K \in(1,2)$,

$$
\lambda^{\mathrm{K}}=\frac{\mathrm{K}(\mathrm{K}-1)}{\Gamma(2-\mathrm{K})} \int_{0}^{\infty}\left(\mathrm{e}^{-x \lambda}-1+x \lambda\right) x^{-1-\mathrm{K}} \mathrm{d} x,
$$

it is easy to calculate the covariance

$$
R_{X, 0}^{(\mathrm{K})}(s, t):=\operatorname{cov}\left(X_{0}^{(\mathrm{K})}(s), X_{0}^{(\mathrm{K})}(t)\right) .
$$

We have

$$
R_{X, 0}^{(\mathrm{K})}(s, t)= \begin{cases}\frac{\Gamma(1-\mathrm{K})}{\mathrm{K}}\left(s^{\mathrm{K}}+t^{\mathrm{K}}-(s+t)^{\mathrm{K}}\right), & \mathrm{K} \in(0,1), \\ \ln s+\ln t-\ln (s+t), & \mathrm{K}=1, \\ \frac{\Gamma(2-\mathrm{K})}{\mathrm{K}(\mathrm{K}-1)}\left(-s^{\mathrm{K}}-t^{\mathrm{K}}+(s+t)^{\mathrm{K}}\right), & \mathrm{K} \in(1,2) .\end{cases}
$$


Next, we rescale time by introducing a process

$$
X_{0}^{(\mathrm{H}, \mathrm{K})}(t):=X_{0}^{(\mathrm{K})}\left(|t|^{2 \mathrm{H}}\right), \quad t \in \mathbb{R},
$$

which has the covariance

$$
R_{X, 0}^{(\mathrm{H}, \mathrm{K})}(s, t):=\operatorname{cov}\left(X_{0}^{(\mathrm{H}, \mathrm{K})}(s), X_{0}^{(\mathrm{H}, \mathrm{K})}(t)\right)
$$

given by

$$
R_{X, 0}^{(\mathrm{H}, \mathrm{K})}(s, t)= \begin{cases}\frac{\Gamma(1-\mathrm{K})}{\mathrm{K}}\left(|s|^{2 \mathrm{HK}}+|t|^{2 \mathrm{HK}}-\left(|s|^{2 \mathrm{H}}+|t|^{2 \mathrm{H}}\right)^{\mathrm{K}}\right), & \mathrm{K} \in(0,1), \\ 2 \mathrm{H}(\ln |s|+\ln |t|-\ln (|s|+|t|)), & \mathrm{K}=1, \\ \frac{\Gamma(2-\mathrm{K})}{\mathrm{K}(\mathrm{K}-1)}\left(-|s|^{2 \mathrm{HK}}-|t|^{2 \mathrm{HK}}+\left(|s|^{2 \mathrm{H}}+|t|^{2 \mathrm{H}}\right)^{\mathrm{K}}\right), & \mathrm{K} \in(1,2) .\end{cases}
$$

If $\mathrm{HK} \leq 1$, consider the usual $\mathrm{fBm} W^{(\mathrm{HK})}(t), t \in \mathbb{R}$, with covariance from (1.1),

$$
R_{W}^{(\mathrm{HK})}(s, t)=\frac{1}{2}\left(|s|^{2 \mathrm{HK}}+|t|^{2 \mathrm{HK}}-|t-s|^{2 \mathrm{HK}}\right)
$$

and, for $\mathrm{K} \in(1,2)$, obtain bfBm just by adding up the independent processes

$$
\mathbf{B}^{(\mathrm{H}, \mathrm{K})}(t):=\sqrt{\frac{\mathrm{K}(\mathrm{K}-1)}{2^{\mathrm{K}} \Gamma(2-\mathrm{K})}} X_{0}^{(\mathrm{H}, \mathrm{K})}(t)+\sqrt{2^{1-\mathrm{K}}} W^{(\mathrm{HK})}(t), \quad t \in \mathbb{R} .
$$

For the boundary case $\mathrm{K}=2$ the integral representation (2.3) does not work but we may simply define $X_{0}^{(2)}(t), t \geq 0$, as a degenerated random linear process with covariance

$$
R_{X, 0}^{(2)}(s, t):=\operatorname{cov}\left(X_{0}^{(2)}(s), X_{0}^{(2)}(t)\right)=2 s t=\left(-s^{2}-t^{2}+(s+t)^{2}\right),
$$

then let again $X_{0}^{(\mathrm{H}, 2)}(t):=X_{0}^{(2)}\left(|t|^{2 \mathrm{H}}\right), t \in \mathbb{R}$, as in (2.4), and obtain

$$
\mathbf{B}^{(\mathrm{H}, 2)}(t):=2^{-1} X_{0}^{(\mathrm{H}, 2)}(t)+2^{-1 / 2} W^{(2 \mathrm{H})}(t), \quad t \in \mathbb{R},
$$

whenever $0<\mathrm{H} \leq \frac{1}{2}$.

In another adjacent case $\mathrm{K}=1$ the $\mathrm{bfBm} \mathbf{B}^{(\mathrm{H}, 1)}$ reduces to the classical $\mathrm{fBm} W^{(\mathrm{H})}$. We hesitate to call it a boundary case because it separates not the zones of existence and non-existence but rather two existence zones with different properties.

In the zone $0<\mathrm{K}<1$ the representation (2.5) does not work because the signs in the covariance of $X^{(\mathrm{K})}$ are opposite to the desired ones. In exchange, we have a representation for fBm

$$
W^{(\mathrm{HK})}(t):=\sqrt{\frac{\mathrm{K}}{2 \Gamma(1-\mathrm{K})}} X_{0}^{(\mathrm{H}, \mathrm{K})}(t)+\sqrt{2^{\mathrm{K}-1}} \mathbf{B}^{(\mathrm{H}, \mathrm{K})}(t), \quad t \in \mathbb{R},
$$

with independent processes on the right hand side. This is equivalent to

$$
\mathbf{B}^{(\mathrm{H}, \mathrm{K})}(t)=\sqrt{2^{1-\mathrm{K}}} W^{(\mathrm{HK})}(t)-\sqrt{\frac{\mathrm{K}}{2^{\mathrm{K}} \Gamma(1-\mathrm{K})}} X_{0}^{(\mathrm{H}, \mathrm{K})}(t), \quad t \in \mathbb{R} .
$$

Since $X_{0}^{(\mathrm{K})}(\cdot)$ is a smooth process, it becomes obvious that the local properties of $\mathbf{B}^{(\mathrm{H}, \mathrm{K})}(\cdot)$ are the same as those of $\mathrm{fBm} W^{(\mathrm{HK})}(\cdot), c f .[18,19]$.

We also see that if $0<\mathrm{K}<1$ and $\mathbf{B}^{(\mathrm{H}, \mathrm{K})}(\cdot)$ exists, then $B^{(\mathrm{HK})}(\cdot)$ exists [7], which simply means $\mathrm{HK} \leq 1$. In Proposition 3.1 we show that $\mathrm{HK} \leq 1$ is necessary for the existence of $\mathbf{B}^{(\mathrm{H}, \mathrm{K})}(\cdot)$ whatever $\mathrm{K}$ is. 
In the following we prefer to work with a modification of the processes $X_{0}^{(\mathrm{K})}, X_{0}^{(\mathrm{H}, \mathrm{K})}$ having simpler covariances. For $\mathrm{K} \in(0,1), \mathrm{H}>0$, let

$$
\begin{aligned}
X^{(\mathrm{K})}(t) & :=\sqrt{\frac{\mathrm{K}}{\Gamma(1-\mathrm{K})}} X_{0}^{(\mathrm{K})}(t), \\
X^{(\mathrm{H}, \mathrm{K})}(t) & :=\sqrt{\frac{\mathrm{K}}{\Gamma(1-\mathrm{K})}} X_{0}^{(\mathrm{H}, \mathrm{K})}(t) .
\end{aligned}
$$

The respective covariances are

$$
\begin{aligned}
R_{X}^{(\mathrm{K})}(s, t) & =\frac{\mathrm{K}}{\Gamma(1-\mathrm{K})} R_{X, 0}^{(\mathrm{K})}(s, t)=s^{\mathrm{K}}+t^{\mathrm{K}}-(s+t)^{\mathrm{K}}, \quad s, t \geq 0, \\
R_{X}^{(\mathrm{H}, \mathrm{K})}(s, t) & =\frac{\mathrm{K}}{\Gamma(1-\mathrm{K})} R_{X, 0}^{(\mathrm{H}, \mathrm{K})}(s, t) \\
& =|s|^{2 \mathrm{HK}}+|t|^{2 \mathrm{HK}}-\left(|s|^{2 \mathrm{H}}+|t|^{2 \mathrm{H}}\right)^{\mathrm{K}}, \quad s, t \in \mathbb{R} .
\end{aligned}
$$

Then (2.6) becomes

$$
\sqrt{2} W^{(\mathrm{HK})}(t)=X^{(\mathrm{H}, \mathrm{K})}(t)+\sqrt{2^{\mathrm{K}}} \mathbf{B}^{(\mathrm{H}, \mathrm{K})}(t), \quad t \in \mathbb{R},
$$

or, in the language of covariances,

$$
2 R_{W}^{(\mathrm{HK})}=R_{X}^{(\mathrm{H}, \mathrm{K})}+2^{\mathrm{K}} R_{\mathrm{B}}^{(\mathrm{H}, \mathrm{K})} .
$$

Finally, notice that an extension to more general processes similar to Proposition 2.1 is also possible for the range $1 \leq \mathrm{K} \leq 2$ (cf. [14], Thm. $3.1(\mathrm{i})$ ).

\section{NECESSARY CONDITIONS}

First of all notice that we must distinguish the existence of bfBm on $\mathbb{R}_{+}$and on $\mathbb{R}$. This is very different from the case of usual $\mathrm{fBm}$ where condition $0<\mathrm{H} \leq 1$ is necessary and sufficient for the existence in both cases.

Proposition 3.1. If bfBm exists on $\mathbb{R}_{+}$, then $\mathrm{K} \leq 2$ and $\mathrm{HK} \leq 1$.

Proof. Since the covariance $R_{\mathrm{B}}^{(\mathrm{H}, \mathrm{K})}(\cdot, \cdot)$ has the self-similarity property

$$
R_{\mathrm{B}}^{(\mathrm{H}, \mathrm{K})}(c s, c t)=c^{2 \mathrm{HK}} R_{\mathrm{B}}^{(\mathrm{H}, \mathrm{K})}(s, t),
$$

we may transform bfBm $\mathbf{B}^{(\mathrm{H}, \mathrm{K})}$ into a stationary process by letting

$$
U_{\mathbf{B}}^{(\mathrm{H}, \mathrm{K})}(\tau):=\mathrm{e}^{-\mathrm{HK} \tau} \mathbf{B}^{(\mathrm{H}, \mathrm{K})}\left(\mathrm{e}^{\tau}\right) .
$$

Stationarity of $U_{\mathbf{B}}^{(\mathrm{H}, \mathrm{K})}$ means that its covariance function depends only on the arguments' difference, i.e.

$$
\operatorname{cov}\left(U_{\mathbf{B}}^{(\mathrm{H}, \mathrm{K})}\left(\tau_{1}\right), U_{\mathbf{B}}^{(\mathrm{H}, \mathrm{K})}\left(\tau_{2}\right)\right)=: \tilde{R}_{\mathbf{B}}^{(\mathrm{H}, \mathrm{K})}\left(\tau_{2}-\tau_{1}\right)
$$

where in our case

$$
\begin{aligned}
\tilde{R}_{\mathrm{B}}^{(\mathrm{H}, \mathrm{K})}(\tau) & =\mathrm{e}^{-\mathrm{HK} \tau} R_{\mathrm{B}}^{(\mathrm{H}, \mathrm{K})}\left(1, \mathrm{e}^{\tau}\right) \\
& =\mathrm{e}^{-\mathrm{HK} \tau} 2^{-\mathrm{K}}\left(\left(1+\mathrm{e}^{2 \mathrm{H} \tau}\right)^{\mathrm{K}}-\left|\mathrm{e}^{\tau}-1\right|^{2 \mathrm{HK}}\right) \\
& =(\cosh (\mathrm{H} \tau))^{\mathrm{K}}-2^{(2 \mathrm{H}-1) \mathrm{K}}|\sinh (\tau / 2)|^{2 \mathrm{HK}} .
\end{aligned}
$$


By Hölder's inequality

$$
\begin{aligned}
\left|\tilde{R}_{\mathrm{B}}^{(\mathrm{H}, \mathrm{K})}(\tau)\right| & =\left|\operatorname{cov}\left(U_{\mathbf{B}}^{(\mathrm{H}, \mathrm{K})}(0), U_{\mathbf{B}}^{(\mathrm{H}, \mathrm{K})}(\tau)\right)\right| \\
& \leq\left[\mathbb{E} U_{\mathbf{B}}^{(\mathrm{H}, \mathrm{K})}(0)^{2} \mathbb{E} U_{\mathbf{B}}^{(\mathrm{H}, \mathrm{K})}(\tau)^{2}\right]^{1 / 2} \\
& =\left[\tilde{R}_{\mathrm{B}}^{(\mathrm{H}, \mathrm{K})}(0) \cdot \tilde{R}_{\mathrm{B}}^{(\mathrm{H}, \mathrm{K})}(0)\right]^{1 / 2} \\
& =\tilde{R}_{\mathrm{B}}^{(\mathrm{H}, \mathrm{K})}(0)=1,
\end{aligned}
$$

hence, the function $\left|\tilde{R}_{\mathrm{B}}^{(\mathrm{H}, \mathrm{K})}(\cdot)\right|$ must be bounded and must attain its maximum at zero (this is a common property of all stationary processes).

In our case, when $\tau \rightarrow+\infty$, in (3.1) we have the expansions

$$
\begin{aligned}
2^{-\mathrm{K}} \mathrm{e}^{-\mathrm{HK} \tau}\left(1+\mathrm{e}^{2 \mathrm{H} \tau}\right)^{\mathrm{K}} & =2^{-\mathrm{K}} \mathrm{e}^{\mathrm{HK} \tau}\left(1+\mathrm{e}^{-2 \mathrm{H} \tau}\right)^{\mathrm{K}} \\
& =2^{-\mathrm{K}} \mathrm{e}^{\mathrm{HK} \tau}\left(1+\mathrm{Ke}^{-2 \mathrm{H} \tau}(1+o(1))\right) \\
& =2^{-\mathrm{K}} \mathrm{e}^{\mathrm{HK} \tau}+2^{-\mathrm{K}} \mathrm{Ke}^{\mathrm{H}(\mathrm{K}-2) \tau}(1+o(1))
\end{aligned}
$$

and

$$
\begin{aligned}
2^{-\mathrm{K}} \mathrm{e}^{-\mathrm{HK} \tau}\left(\mathrm{e}^{\tau}-1\right)^{2 \mathrm{HK}} & =2^{-\mathrm{K}} \mathrm{e}^{\mathrm{HK} \tau}\left(1-\mathrm{e}^{-\tau}\right)^{2 \mathrm{HK}} \\
& =2^{-\mathrm{K}} \mathrm{e}^{\mathrm{HK} \tau}\left(1-2 \mathrm{HKe}^{-\tau}(1+o(1))\right) \\
& =2^{-\mathrm{K}} \mathrm{e}^{\mathrm{HK} \tau}-\mathrm{HK}^{1-\mathrm{K}} \mathrm{e}^{(\mathrm{HK}-1) \tau}(1+o(1))
\end{aligned}
$$

that yields

$$
\tilde{R}_{\mathrm{B}}^{(\mathrm{H}, \mathrm{K})}(\tau)=2^{-\mathrm{K}} \mathrm{Ke}^{(\mathrm{K}-2) \mathrm{H} \tau}(1+o(1))+\mathrm{HK} 2^{1-\mathrm{K}} \mathrm{e}^{(\mathrm{HK}-1) \tau}(1+o(1)) .
$$

Therefore, the boundedness of $\tilde{R}(\cdot)$ implies that both conditions $\mathrm{K} \leq 2$ and $\mathrm{HK} \leq 1$ are necessary for the existence of $\mathbf{B}^{(\mathrm{H}, \mathrm{K})}$ on $\mathbb{R}_{+}$.

Another argument for $\mathrm{HK} \leq 1$ is given in $([14]$, p. 626).

Proposition 3.2. The following two covariance based necessary conditions hold.

(a) If bfBm exists on $\mathbb{R}$, then $\mathrm{K} \leq \frac{1}{2 \mathrm{H}-1}$.

(b) If bfBm exists on $\mathbb{R}_{+}$, then $\mathrm{K} \leq \widehat{\mathrm{K}}(\mathrm{H})$, where

$$
\widehat{\mathrm{K}}(\mathrm{H}):=\sup \left\{\mathrm{K}: \sup _{\tau>0}\left((\cosh (\mathrm{H} \tau))^{\mathrm{K}}-2^{(2 \mathrm{H}-1) \mathrm{K}}|\sinh (\tau / 2)|^{2 \mathrm{HK}}\right) \leq 1\right\}
$$

and $\widehat{\mathrm{K}}(\mathrm{H})<\mathrm{H}^{-1}$ for $\mathrm{H}>1$.

Remark 3.3. We do not have an analytic expression for the function $\widehat{\mathrm{K}}(\cdot)$. Some values of $\widehat{\mathrm{K}}(\cdot)$ are given in Table 1 below.

\section{Proof.}

(a) Assume that bfBm exists on $\mathbb{R}$. Since for its covariance we have $R_{\mathrm{B}}^{(\mathrm{H}, \mathrm{K})}(1,1)=R_{\mathrm{B}}^{(\mathrm{H}, \mathrm{K})}(-1,-1)=1$, it is true that

$$
-1 \leq R_{\mathrm{B}}^{(\mathrm{H}, \mathrm{K})}(1,-1)=2^{-\mathrm{K}}\left[2^{\mathrm{K}}-2^{2 \mathrm{HK}}\right]=1-2^{(2 \mathrm{H}-1) \mathrm{K}},
$$

whereas $(2 \mathrm{H}-1) \mathrm{K} \leq 1$. 
TABLE 1. Existence boundary $\overline{\mathrm{K}}(\mathrm{H})$ and its covariance upper bound $\widehat{\mathrm{K}}(\mathrm{H})$.

\begin{tabular}{ccccccccc}
\hline $\mathrm{H}$ & $\overline{\mathrm{K}}(\mathrm{H})$ & $\widehat{\mathrm{K}}(\mathrm{H})$ & $\mathrm{H}$ & $\overline{\mathrm{K}}(\mathrm{H})$ & $\widehat{\mathrm{K}}(\mathrm{H})$ & $\mathrm{H}$ & $\overline{\mathrm{K}}(\mathrm{H})$ & $\widehat{\mathrm{K}}(\mathrm{H})$ \\
\hline 1.01 & 0.988 & 0.988 & 2 & 0.422 & 0.440 & 6 & 0.117 & 0.123 \\
\hline 1.1 & 0.887 & 0.894 & 2.5 & 0.321 & 0.338 & 7 & 0.099 & 0.104 \\
\hline 1.2 & 0.794 & 0.807 & 3 & 0.260 & 0.273 & 10 & 0.067 & 0.070 \\
\hline 1.3 & 0.718 & 0.734 & 3.5 & 0.217 & 0.228 & 20 & 0.032 & 0.033 \\
\hline 1.5 & 0.603 & 0.619 & 4 & 0.185 & 0.196 & 60 & 0.010 & 0.010 \\
\hline 1.7 & 0.519 & 0.533 & 5 & 0.144 & 0.152 & 100 & 0.006 & 0.006 \\
\hline
\end{tabular}

(b) Assume that bfBm exists on $\mathbb{R}_{+}$. Then the stationary process $U_{\mathbf{B}}^{\mathrm{H}, \mathrm{K}}$ with covariance $\tilde{R}_{\mathrm{B}}^{(\mathrm{H}, \mathrm{K})}$ exists. Then $(3.2)$ yields

$$
\sup _{\tau>0}\left((\cosh (\mathrm{H} \tau))^{\mathrm{K}}-2^{(2 \mathrm{H}-1) \mathrm{K}}(\sinh (\tau / 2))^{2 \mathrm{HK}}\right) \leq 1
$$

which is equivalent to

$$
(2 \cosh (\mathrm{H} \tau))^{\mathrm{K}} \leq(2 \sinh (\tau / 2))^{2 \mathrm{HK}}+2^{\mathrm{K}}, \quad \tau \geq 0 .
$$

It remains to notice that if (3.4) holds for some value of $\mathrm{K}$, then it holds for any smaller positive value of $\mathrm{K}$, since for any $a \in(0,1]$ we have

$$
(2 \cosh (\mathrm{H} \tau))^{a \mathrm{~K}} \leq\left[(2 \sinh (\tau / 2))^{2 \mathrm{HK}}+2^{\mathrm{K}}\right]^{a} \leq(2 \sinh (\tau / 2))^{2 \mathrm{H} a \mathrm{~K}}+2^{a \mathrm{~K}} .
$$

Finally, if $\mathrm{H}>1$ and $\mathrm{K}=\mathrm{H}^{-1}$, then

$$
\lim _{\tau \rightarrow \infty}\left((\cosh (\mathrm{H} \tau))^{\mathrm{K}}-2^{(2 \mathrm{H}-1) \mathrm{K}}(\sinh (\tau / 2))^{2 \mathrm{HK}}\right)=2^{1-\mathrm{K}}>1 .
$$

Hence, (3.3) fails for $\mathrm{K}=\mathrm{H}^{-1}$. Moreover, by continuity arguments, it also fails for all $\mathrm{K}$ that are sufficiently close to $\mathrm{H}^{-1}$. It follows that $\widehat{\mathrm{K}}(\mathrm{H})<\mathrm{H}^{-1}$.

The covariance criteria given in this section are quite elementary. They take into account only 2-dimensional distributions of the process. In order to get sharper results, we need more refined spectral methods.

\section{Spectral analysis}

\subsection{Stationary processes, covariances and spectral densities}

In addition to the self-similar processes $W^{(\mathrm{H})}, X^{(\mathrm{K})}, X^{(\mathrm{H}, \mathrm{K})}, \mathbf{B}^{(\mathrm{H}, \mathrm{K})}$, let us introduce their stationary versions

$$
\begin{aligned}
U_{W}^{(\mathrm{H})}(\tau) & :=\mathrm{e}^{-\mathrm{H} \tau} W^{(\mathrm{H})}\left(\mathrm{e}^{\tau}\right) ; \\
U_{X}^{(\mathrm{K})}(\tau) & :=\mathrm{e}^{-\mathrm{K} \tau / 2} X^{(\mathrm{K})}\left(\mathrm{e}^{\tau}\right) ; \\
U_{X}^{(\mathrm{H}, \mathrm{K})}(\tau) & :=\mathrm{e}^{-\mathrm{HK} \tau} X^{(\mathrm{H}, \mathrm{K})}\left(\mathrm{e}^{\tau}\right) ; \\
U_{\mathbf{B}}^{(\mathrm{H}, \mathrm{K})}(\tau) & :=\mathrm{e}^{-\mathrm{HK} \tau} \mathbf{B}^{(\mathrm{H}, \mathrm{K})}\left(\mathrm{e}^{\tau}\right) .
\end{aligned}
$$

Notice that $U_{W}^{(\mathrm{H})}$ is one of the well known versions of fractional Ornstein-Uhlenbeck process (see e.g. [3,5]). By the definition of $X^{(\mathrm{H}, \mathrm{K})}$, we also have

$$
\begin{aligned}
U_{X}^{(\mathrm{H}, \mathrm{K})}(\tau) & =\mathrm{e}^{-\mathrm{HK} \tau} X^{(\mathrm{K})}\left(\left(\mathrm{e}^{\tau}\right)^{2 \mathrm{H}}\right) \\
& =\mathrm{e}^{-\mathrm{K}(2 \mathrm{H} \tau) / 2} X^{(\mathrm{K})}\left(\mathrm{e}^{2 \mathrm{H} \tau}\right)=U_{X}^{(\mathrm{K})}(2 \mathrm{H} \tau) .
\end{aligned}
$$


The covariance functions corresponding to these four stationary processes are

$$
\begin{aligned}
\tilde{R}_{W}^{(\mathrm{H})}(\tau) & :=\cosh (\mathrm{H} \tau)-2^{2 \mathrm{H}-1}|\sinh (\tau / 2)|^{2 \mathrm{H}} ; \\
\tilde{R}_{X}^{(\mathrm{K})}(\tau) & :=2 \cosh (\mathrm{K} \tau / 2)-(2 \cosh (\tau / 2))^{\mathrm{K}} ; \\
\tilde{R}_{X}^{(\mathrm{H}, \mathrm{K})}(\tau) & :=\tilde{R}_{X}^{(\mathrm{K})}(2 \mathrm{H} \tau)=2 \cosh (\mathrm{HK} \tau)-(2 \cosh (\mathrm{H} \tau))^{\mathrm{K}} ; \\
\tilde{R}_{\mathrm{B}}^{(\mathrm{H}, \mathrm{K})}(\tau) & :=(\cosh (\mathrm{H} \tau))^{\mathrm{K}}-2^{(2 \mathrm{H}-1) \mathrm{K}}|\sinh (\tau / 2)|^{2 \mathrm{HK}} .
\end{aligned}
$$

The basic equality (2.9) transforms into

$$
2 \tilde{R}_{W}^{(\mathrm{HK})}(\tau)=\tilde{R}_{X}^{(\mathrm{H}, \mathrm{K})}(\tau)+2^{\mathrm{K}} \tilde{R}_{\mathbf{B}}^{(\mathrm{H}, \mathrm{K})}(\tau) .
$$

Let us now pass to spectral representations. Recall that by inversion formula any covariance function $\tilde{R}(\cdot)$ of a stationary process such that $\tilde{R} \in L_{1}(\mathbb{R})$ admits a spectral representation

$$
\tilde{R}(\tau)=\int_{-\infty}^{\infty} \mathrm{e}^{i \tau u} f(u) \mathrm{d} u, \quad \tau \in \mathbb{R},
$$

and the non-negative summable function $f(\cdot)$ is called the spectral density of the corresponding process. We denote $f_{W}^{(\mathrm{H})}, f_{X}^{(\mathrm{K})}, f_{X}^{(\mathrm{H}, \mathrm{K})}, f_{\mathrm{B}}^{(\mathrm{H}, \mathrm{K})}$ the spectral densities corresponding to the respective covariance functions defined above.

Notice immediately that relation $\tilde{R}_{X}^{(\mathrm{H}, \mathrm{K})}(\tau)=\tilde{R}_{X}^{(\mathrm{K})}(2 \mathrm{H} \tau)$ yields

$$
f_{X}^{(\mathrm{H}, \mathrm{K})}(u)=\frac{1}{2 \mathrm{H}} f_{X}^{(\mathrm{K})}\left(\frac{u}{2 \mathrm{H}}\right) .
$$

\subsection{Spectral criterion for the existence of $\mathrm{B}^{(H, K)}$}

Proposition 4.1. Let $\mathrm{K} \in(0,1), \mathrm{H}>0$. Then bfBm $\mathbf{B}^{(\mathrm{H}, \mathrm{K})}$ exists on $\mathbb{R}_{+}$iff

$$
f_{X}^{(\mathrm{H}, \mathrm{K})}(u) \leq 2 f_{W}^{(\mathrm{HK})}(u), \quad u \in \mathbb{R} .
$$

Proof.

(a) Assume that (4.4) holds. Then by (4.2)

$$
\begin{aligned}
2^{\mathrm{K}} \tilde{R}_{\mathrm{B}}^{(\mathrm{H}, \mathrm{K})}(\tau) & =2 \tilde{R}_{W}^{(\mathrm{HK})}(\tau)-\tilde{R}_{X}^{(\mathrm{H}, \mathrm{K})}(\tau) \\
& =\int_{-\infty}^{\infty} \mathrm{e}^{i \tau u}\left(2 f_{W}^{(\mathrm{HK})}(u)-f_{X}^{(\mathrm{H}, \mathrm{K})}(u)\right) \mathrm{d} u \\
& :=\int_{-\infty}^{\infty} \mathrm{e}^{i \tau u} f(u) \mathrm{d} u,
\end{aligned}
$$

where $f(\cdot)$ is a nonnegative integrable function. It follows that a stationary process $U_{\mathbf{B}}^{(\mathrm{H}, \mathrm{K})}(\tau), \tau \in \mathbb{R}$, with covariance $\tilde{R}_{\mathrm{B}}^{(\mathrm{H}, \mathrm{K})}(\cdot)$ exists, and we obtain bfBm by letting

$$
\mathbf{B}^{(\mathrm{H}, \mathrm{K})}(t):=t^{\mathrm{HK}} U_{\mathbf{B}}^{(\mathrm{H}, \mathrm{K})}(\ln t), \quad t \geq 0 .
$$

(b) Conversely, if a bfBm $\mathbf{B}^{\mathrm{H}, \mathrm{K}}$ exists on $\mathbb{R}_{+}$, then a stationary process $U_{\mathbf{B}}^{(\mathrm{H}, \mathrm{K})}(\tau), \tau \in \mathbb{R}$, with covariance $\tilde{R}_{\mathrm{B}}^{(\mathrm{H}, \mathrm{K})}(\cdot)$ exists. Since this function belongs to $L_{1}(\mathbb{R})$, there exists a non-negative spectral density $f$ such that

$$
\tilde{R}_{\mathrm{B}}^{(\mathrm{H}, \mathrm{K})}(\tau)=\int_{-\infty}^{\infty} \mathrm{e}^{i \tau u} f(u) \mathrm{d} u, \quad \tau \in \mathbb{R} .
$$


By (4.2) it follows that for any $\tau \in \mathbb{R}$

$$
\int_{-\infty}^{\infty} \mathrm{e}^{i \tau u}\left(2 f_{W}^{(\mathrm{HK})}-f_{X}^{(\mathrm{H}, \mathrm{K})}-2^{\mathrm{K}} f\right)(u) \mathrm{d} u=2 \tilde{R}_{W}^{(\mathrm{HK})}(\tau)-\tilde{R}_{X}^{(\mathrm{H}, \mathrm{K})}(\tau)-2^{\mathrm{K}} \tilde{R}_{\mathrm{B}}^{(\mathrm{H}, \mathrm{K})}(\tau)=0 .
$$

Since the kernel of Fourier transform is trivial, we have

$$
2 f_{W}^{(\mathrm{HK})}-f_{X}^{(\mathrm{H}, \mathrm{K})}-2^{\mathrm{K}} f=0,
$$

Hence,

$$
2 f_{W}^{(\mathrm{HK})}-f_{X}^{(\mathrm{H}, \mathrm{K})}=2^{\mathrm{K}} f \geq 0 .
$$

The criterion of Proposition 4.1 becomes meaningful whenever we have explicit formulae for the involved spectral densities. They are found below in this section.

\subsection{Spectrum associated to the Lei-Nualart process $X$}

By using the representation (2.3), we obtain

$$
\begin{aligned}
U_{X}^{(\mathrm{K})}(\tau) & =\mathrm{e}^{-\mathrm{K} \tau / 2} X^{(\mathrm{K})}\left(\mathrm{e}^{\tau}\right)=\mathrm{e}^{-\mathrm{K} \tau / 2} \sqrt{\frac{\mathrm{K}}{\Gamma(1-\mathrm{K})}} X_{0}^{(\mathrm{K})}\left(\mathrm{e}^{\tau}\right) \\
& =\sqrt{\frac{\mathrm{K}}{\Gamma(1-\mathrm{K})}} \int_{0}^{\infty} \mathrm{e}^{-\mathrm{K} \tau / 2}\left(1-\mathrm{e}^{-r \mathrm{e}^{\tau}}\right) r^{-(1+\mathrm{K}) / 2} \mathcal{W}(\mathrm{d} r) .
\end{aligned}
$$

It follows that

$$
\begin{aligned}
\tilde{R}_{X}^{(\mathrm{K})}(\tau) & =\operatorname{cov}\left(U_{X}^{(\mathrm{K})}(\tau), U_{X}^{(\mathrm{K})}(0)\right) \\
& =\frac{\mathrm{K}}{\Gamma(1-\mathrm{K})} \int_{0}^{\infty} \mathrm{e}^{-\mathrm{K} \tau / 2}\left(1-\mathrm{e}^{-r \mathrm{e}^{\tau}}\right)\left(1-\mathrm{e}^{-r}\right) r^{-(1+\mathrm{K})} \mathrm{d} r \\
& =\frac{\mathrm{K}}{\Gamma(1-\mathrm{K})} \int_{-\infty}^{\infty} \mathrm{e}^{-\mathrm{K} \tau / 2}\left(1-\mathrm{e}^{-\mathrm{e}^{(v+\tau)}}\right)\left(1-\mathrm{e}^{-\mathrm{e}^{v}}\right) \mathrm{e}^{-\mathrm{K} v} \mathrm{~d} v \\
& =\frac{\mathrm{K}}{\Gamma(1-\mathrm{K})} \int_{-\infty}^{\infty} \mathrm{e}^{-\mathrm{K}(v+\tau) / 2}\left(1-\mathrm{e}^{-\mathrm{e}^{(v+\tau)}}\right) \mathrm{e}^{-\mathrm{K} v / 2}\left(1-\mathrm{e}^{-\mathrm{e}^{v}}\right) \mathrm{d} v \\
& =\frac{\mathrm{K}}{\Gamma(1-\mathrm{K})} \int_{-\infty}^{\infty} g(v+\tau) g(v) \mathrm{d} v,
\end{aligned}
$$

where $g(v):=\mathrm{e}^{-\mathrm{K} v / 2}\left(1-\mathrm{e}^{-\mathrm{e}^{v}}\right)$. By applying Fourier transform, we obtain

$$
\begin{aligned}
\tilde{R}_{X}^{(\mathrm{K})}(\tau) & =\frac{\mathrm{K}}{\Gamma(1-\mathrm{K})} \int_{-\infty}^{\infty} \mathrm{e}^{i \tau u} \widehat{g}(u) \overline{\widehat{g}(u)} \mathrm{d} u \\
& =\frac{\mathrm{K}}{\Gamma(1-\mathrm{K})} \int_{-\infty}^{\infty} \mathrm{e}^{i \tau u}|\widehat{g}(u)|^{2} \mathrm{~d} u .
\end{aligned}
$$

It follows that

$$
f_{X}^{(\mathrm{K})}(u)=\frac{\mathrm{K}}{\Gamma(1-\mathrm{K})}|\widehat{g}(u)|^{2} .
$$


Now we find $\widehat{g}(u)$. By definition

$$
\begin{aligned}
\widehat{g}(u) & =\frac{1}{\sqrt{2 \pi}} \int_{-\infty}^{\infty} \mathrm{e}^{-i u v} g(v) \mathrm{d} v \\
& =\frac{1}{\sqrt{2 \pi}} \int_{-\infty}^{\infty} \mathrm{e}^{-i u v} \mathrm{e}^{-\mathrm{K} v / 2}\left(1-\mathrm{e}^{-\mathrm{e}^{v}}\right) \mathrm{d} v \\
& =\frac{1}{\sqrt{2 \pi}} \int_{0}^{\infty} r^{-i u-\mathrm{K} / 2-1}\left(1-\mathrm{e}^{-r}\right) \mathrm{d} r \\
& =-\frac{1}{\sqrt{2 \pi}(-i u-\mathrm{K} / 2)} \int_{0}^{\infty} r^{-i u-\mathrm{K} / 2} \mathrm{e}^{-r} \mathrm{~d} r \\
& =-\frac{\Gamma(-i u-\mathrm{K} / 2+1)}{\sqrt{2 \pi}(-i u-\mathrm{K} / 2)}=-\frac{\Gamma(-i u-\mathrm{K} / 2)}{\sqrt{2 \pi}} .
\end{aligned}
$$

We conclude that

$$
f_{X}^{(\mathrm{K})}(u)=\frac{\mathrm{K}}{\Gamma(1-\mathrm{K})} \frac{|\Gamma(-i u-\mathrm{K} / 2)|^{2}}{2 \pi}
$$

Finally, equation (4.3) yields

$$
f_{X}^{(\mathrm{H}, \mathrm{K})}(u)=\frac{1}{2 \mathrm{H}} \frac{\mathrm{K}}{\Gamma(1-\mathrm{K})} \frac{\left|\Gamma\left(\frac{-i u}{2 \mathrm{H}}-\mathrm{K} / 2\right)\right|^{2}}{2 \pi} .
$$

\subsection{Spectrum of the fractional Ornstein-Uhlenbeck process}

The layout of calculation is very much the same as for the spectrum of Lei-Nualart process. Recall that fractional Brownian motion, as a process with stationary increments, admits, for $\mathrm{H} \in(0,1)$, a spectral representation

$$
W^{(\mathrm{H})}(t)=\int_{-\infty}^{\infty} \frac{y_{\mathrm{H}}\left(\mathrm{e}^{i t r}-1\right)}{|r|^{\mathrm{H}+1 / 2}} \mathcal{W}(\mathrm{d} r),
$$

where

$$
y_{\mathrm{H}}^{2}=\frac{\Gamma(2 \mathrm{H}+1) \sin (\pi \mathrm{H})}{2 \pi} .
$$

Therefore,

$$
U_{W}^{(\mathrm{H})}(\tau)=\mathrm{e}^{-\mathrm{H} \tau} W^{(\mathrm{H})}\left(\mathrm{e}^{\tau}\right)=\int_{-\infty}^{\infty} \mathrm{e}^{-\mathrm{H} \tau} \frac{y_{\mathrm{H}}\left(\mathrm{e}^{i \mathrm{e}^{\tau} r}-1\right)}{|r|^{\mathrm{H}+1 / 2}} \mathcal{W}(\mathrm{d} r),
$$

and

$$
\begin{aligned}
\tilde{R}_{W}^{(\mathrm{H})}(\tau) & =y_{\mathrm{H}}^{2} \int_{-\infty}^{\infty} \mathrm{e}^{-\mathrm{H} \tau} \frac{\left(\mathrm{e}^{i \mathrm{e}^{\tau} r}-1\right)}{|r|^{\mathrm{H}+1 / 2}} \frac{\overline{\left(\mathrm{e}^{i r}-1\right)}}{|r|^{\mathrm{H}+1 / 2}} \mathrm{~d} r \\
& =2 y_{\mathrm{H}}^{2} \operatorname{Re} \int_{0}^{\infty} \mathrm{e}^{-\mathrm{H} \tau} \frac{\left(\mathrm{e}^{i \mathrm{e}^{\tau} r}-1\right)}{r^{\mathrm{H}+1 / 2}} \frac{\overline{\left(\mathrm{e}^{i r}-1\right)}}{r^{\mathrm{H}+1 / 2}} \mathrm{~d} r \\
& =2 y_{\mathrm{H}}^{2} \operatorname{Re} \int_{-\infty}^{\infty} \mathrm{e}^{-\mathrm{H}(v+\tau)}\left(\mathrm{e}^{i \mathrm{e}^{v+\tau}}-1\right) \overline{\mathrm{e}^{-\mathrm{H} v}\left(\mathrm{e}^{i \mathrm{e}^{v}}-1\right)} \mathrm{d} v \\
& =2 y_{\mathrm{H}}^{2} \operatorname{Re} \int_{-\infty}^{\infty} g(v+\tau) \overline{g(v)} \mathrm{d} v
\end{aligned}
$$

where $g(v):=\mathrm{e}^{-\mathrm{H} v}\left(\mathrm{e}^{i \mathrm{e}^{v}}-1\right)$. 
By applying Fourier transform, we obtain

$$
\begin{aligned}
\tilde{R}_{W}^{(\mathrm{H})}(\tau) & =2 y_{\mathrm{H}}^{2} \operatorname{Re} \int_{-\infty}^{\infty} \mathrm{e}^{i \tau u} \widehat{g}(u) \overline{\widehat{g}(u)} \mathrm{d} u \\
& =2 y_{\mathrm{H}}^{2} \operatorname{Re} \int_{-\infty}^{\infty} \mathrm{e}^{i \tau u}|\widehat{g}(u)|^{2} \mathrm{~d} u \\
& =2 y_{\mathrm{H}}^{2} \int_{-\infty}^{\infty} \cos (\tau u)|\widehat{g}(u)|^{2} \mathrm{~d} u \\
& =2 y_{\mathrm{H}}^{2} \int_{-\infty}^{\infty} \cos (\tau u) \frac{|\widehat{g}(u)|^{2}+|\widehat{g}(-u)|^{2}}{2} \mathrm{~d} u \\
& =2 y_{\mathrm{H}}^{2} \int_{-\infty}^{\infty} \mathrm{e}^{i \tau u} \frac{|\widehat{g}(u)|^{2}+|\widehat{g}(-u)|^{2}}{2} \mathrm{~d} u .
\end{aligned}
$$

It follows that

$$
f_{W}^{(\mathrm{H})}(u)=y_{\mathrm{H}}^{2}\left(|\widehat{g}(u)|^{2}+|\widehat{g}(-u)|^{2}\right) .
$$

Now we find $\widehat{g}(u)$. By definition

$$
\begin{aligned}
\widehat{g}(u) & =\frac{1}{\sqrt{2 \pi}} \int_{-\infty}^{\infty} \mathrm{e}^{-i u v} g(v) \mathrm{d} v \\
& =\frac{1}{\sqrt{2 \pi}} \int_{-\infty}^{\infty} \mathrm{e}^{-(i u+\mathrm{H}) v}\left(\mathrm{e}^{i \mathrm{e}^{v}}-1\right) \mathrm{d} v \\
& :=\frac{1}{\sqrt{2 \pi}} \int_{-\infty}^{\infty} \mathrm{e}^{-z v}\left(\mathrm{e}^{i \mathrm{e}^{v}}-1\right) \mathrm{d} v \\
& =\frac{i}{\sqrt{2 \pi} z} \int_{-\infty}^{\infty} \mathrm{e}^{-z v} \mathrm{e}^{v} \mathrm{e}^{i \mathrm{e}^{v}} \mathrm{~d} v \\
& =\frac{i}{\sqrt{2 \pi} z} \int_{0}^{\infty} r^{-z} \mathrm{e}^{i r} \mathrm{~d} r \\
& =\frac{i}{\sqrt{2 \pi} z} \int_{0}^{\infty} r^{-z}(\cos r+i \sin r) \mathrm{d} r \\
& =\frac{i}{\sqrt{2 \pi} z}\left(\frac{\pi}{2 \Gamma(z) \cos (\pi z / 2)}+\frac{i \pi}{2 \Gamma(z) \sin (\pi z / 2)}\right) \\
& =\frac{-\sqrt{\pi / 2}}{z \Gamma(z)} \frac{\cos (\pi z / 2)-i \sin (\pi z / 2)}{\sin (\pi z)} \\
& =\frac{-\sqrt{\pi / 2}}{z \Gamma(z)} \frac{\mathrm{e}^{-i \pi z / 2}}{\sin (\pi z)},
\end{aligned}
$$

where $z=\mathrm{H}+i u$. Notice that the integrals that appear after the integration by parts must be understood as the main value integrals (they are not absolutely converging).

We infer that

$$
|\widehat{g}(u)|^{2}=\frac{\pi / 2}{|z|^{2}|\Gamma(z)|^{2}} \frac{\mathrm{e}^{\pi u}}{|\sin (\pi z)|^{2}} .
$$

By using trigonometric formulae

$$
\begin{aligned}
\sin (\pi z) & =\sin (\pi \mathrm{H}+i \pi u)=\sin (\pi \mathrm{H}) \cos (i \pi u)+\cos (\pi \mathrm{H}) \sin (i \pi u) \\
& =\sin (\pi \mathrm{H}) \cosh (\pi u)+i \cos (\pi \mathrm{H}) \sinh (\pi u), \\
|\sin (\pi z)|^{2} & =\sin ^{2}(\pi \mathrm{H}) \cosh ^{2}(\pi u)+\cos ^{2}(\pi \mathrm{H}) \sinh ^{2}(\pi u),
\end{aligned}
$$


we may conclude that

$$
\begin{aligned}
f_{W}^{(\mathrm{H})}(u) & =y_{\mathrm{H}}^{2} \frac{\pi / 2}{|z|^{2}|\Gamma(z)|^{2}} \frac{\mathrm{e}^{\pi u}+\mathrm{e}^{-\pi u}}{\sin ^{2}(\pi \mathrm{H}) \cosh ^{2}(\pi u)+\cos ^{2}(\pi \mathrm{H}) \sinh ^{2}(\pi u)} \\
& =\frac{\Gamma(2 \mathrm{H}+1) \sin (\pi \mathrm{H})}{2\left(u^{2}+\mathrm{H}^{2}\right)|\Gamma(\mathrm{H}+i u)|^{2}} \frac{\cosh (\pi u)}{\sin ^{2}(\pi \mathrm{H}) \cosh ^{2}(\pi u)+\cos ^{2}(\pi \mathrm{H}) \sinh ^{2}(\pi u)} .
\end{aligned}
$$

An interesting special case is $\mathrm{H}=1 / 2$ where, using a formula 6.1 .30 from [1],

$$
|\Gamma(1 / 2+i u)|^{2}=\frac{\pi}{\cosh (\pi u)},
$$

we get

$$
f_{W}^{(1 / 2)}(u)=\frac{2}{\pi\left(4 u^{2}+1\right)}
$$

in accordance with the classical Ornstein-Uhlenbeck covariance function

$$
\tilde{R}_{W}^{(1 / 2)}(\tau)=\mathrm{e}^{-|\tau| / 2} .
$$

There is an alternative approach to the computation of the spectral density $f_{W}^{(\mathrm{H})}, \mathrm{H} \neq 1 / 2$, due to BarndorffNielsen and Perez-Abreu, [3]. Writing

$$
\tilde{R}_{W}^{(\mathrm{H})}(\tau)=\frac{\mathrm{e}^{\mathrm{H} \tau}}{2}\left(1+\mathrm{e}^{-2 \mathrm{H} \tau}-\left(1-\mathrm{e}^{-\tau}\right)^{2 \mathrm{H}}\right), \quad \tau>0,
$$

and using Taylor expansion

$$
(1-v)^{2 \mathrm{H}}=1+\sum_{k=1}^{\infty}(-1)^{k} C_{k, \mathrm{H}} v^{k}, \quad C_{k, \mathrm{H}}:=\frac{\Gamma(2 \mathrm{H}+1)}{\Gamma(k+1) \Gamma(2 \mathrm{H}-k+1)},
$$

one obtains

$$
\tilde{R}_{W}^{(\mathrm{H})}(\tau)=\frac{\mathrm{e}^{-\mathrm{H}|\tau|}}{2}+\sum_{k=1}^{\infty}(-1)^{k+1} \frac{C_{k, \mathrm{H}}}{2} \mathrm{e}^{-(k-\mathrm{H})|\tau|}, \quad \tau \in \mathbb{R},
$$

whereas

$$
f_{W}^{(\mathrm{H})}(u)=\sum_{k=0}^{\infty}(-1)^{k+1} C_{k, \mathrm{H}} \frac{k-\mathrm{H}}{2 \pi\left(u^{2}+(k-\mathrm{H})^{2}\right)} .
$$

\section{Computation}

According to Proposition 3.2, in order to check the existence of bfBm on $\mathbb{R}_{+}$, one must check for each pair ( $\mathrm{H}, \mathrm{K})$ with $\mathrm{H}>1,0<\mathrm{K}<\mathrm{H}^{-1}$, whether for all $u \geq 0$,

$$
\begin{aligned}
f_{X}^{(\mathrm{H}, \mathrm{K})}(u) & =\frac{1}{2 \mathrm{H}} \frac{\mathrm{K}}{\Gamma(1-\mathrm{K})} \frac{\left|\Gamma\left(\frac{-i u}{2 \mathrm{H}}-\mathrm{K} / 2\right)\right|^{2}}{2 \pi} \\
& \leq 2 f_{W}^{(\mathrm{HK})}(u) \\
& =\frac{\Gamma(2 \mathrm{HK}+1) \sin (\pi \mathrm{HK})}{\left(u^{2}+(\mathrm{HK})^{2}\right)|\Gamma(\mathrm{HK}+i u)|^{2}} \frac{\cosh (\pi u)}{\sin ^{2}(\pi \mathrm{HK}) \cosh ^{2}(\pi u)+\cos ^{2}(\pi \mathrm{HK}) \sinh ^{2}(\pi u)} .
\end{aligned}
$$

Our numerical computations show that for every fixed $\mathrm{H}>1$ there is a positive bound $\overline{\mathrm{K}}$ such that condition (5.1) holds for all $\mathrm{K}<\overline{\mathrm{K}}$ and does not hold for all $\mathrm{K}>\overline{\mathrm{K}}$. 


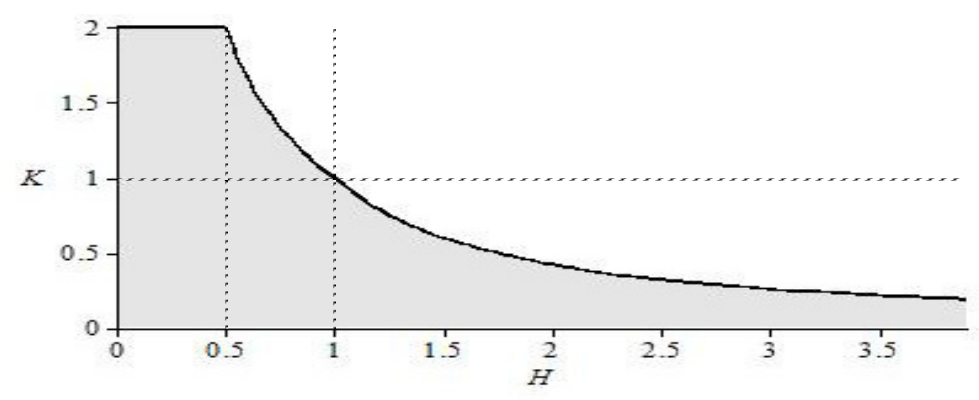

Figure 1. Global zone of existence for $\operatorname{bfBm}\left\{\mathbf{B}^{(\mathrm{H}, \mathrm{K})}(t), t \geq 0\right\}$.

More precisely, we went through the range $\mathrm{H} \in(1,100]$ with a step 0.01 . For every $\mathrm{H}$ we went through the range $\mathrm{K} \in\left(0, \mathrm{H}^{-1}\right)$ with the same step 0.01 and checked inequality $(5.1)$ for $u \in[0,50]$ with the step 0.01 . Computations indicate that larger values of $u$ are by far irrelevant. They also show the existence of the boundary $\overline{\mathrm{K}}:=\overline{\mathrm{K}}(\mathrm{H})$, separating the existence and the non-existence zones as said above. Furthermore, we repeated the procedure with the smaller step 0.001 in $\mathrm{K}$ near the boundary value, in order to get sharper values of function $\overline{\mathrm{K}}$.

Few words about the boundary case: since the expression for covariance function of bfBm is continuous in $H$ and $K$, it is clear that the property of its non-negative definiteness is conserved when we let the parameters go to some limits. In other words, the domain of existence of bfBm is closed on the plane $(H, K)$. In particular, the bfBm with boundary parameters $(H, \bar{K}(H))$ should exist.

A sample of values of $\overline{\mathrm{K}}(\mathrm{H})$ is given in Table 1 along with its upper bound $\widehat{\mathrm{K}}(\mathrm{H})$. The complete table of values of functions $\overline{\mathrm{K}}(\mathrm{H})$ and $\widehat{\mathrm{K}}(\mathrm{H})$ may be found in [12]. The bound $\widehat{\mathrm{K}}(\mathrm{H})$ is rather sharp, especially for large values of $\mathrm{H}$.

The resulting global zone of existence for $\operatorname{bfBm}\left\{\mathbf{B}^{(\mathrm{H}, \mathrm{K})}(t), t \geq 0\right\}$, is represented at Figure 1.

\section{Some BOUndary CASES}

\subsection{A limiting process for $\mathrm{K}=0$}

Let us consider a limiting behavior of the bfBm covariance function when $\mathrm{H}>0$ is fixed and $\mathrm{K} \rightarrow 0$. For $\tau>0$ we have

$$
\begin{aligned}
\mathrm{K}^{-1} \tilde{R}_{\mathrm{B}}^{(\mathrm{H}, \mathrm{K})}(\tau) & =\mathrm{K}^{-1}(\exp (\mathrm{K} \ln (\cosh (\mathrm{H} \tau)))-\exp (\mathrm{K}((2 \mathrm{H}-1) \ln 2+\ln \sinh (\tau / 2)))) \\
& \rightarrow \ln (\cosh (\mathrm{H} \tau))-(2 \mathrm{H}-1) \ln 2-\ln \sinh (\tau / 2) \\
& =\ln (2 \cosh (\mathrm{H} \tau))-2 \mathrm{H} \ln (2 \sinh (\tau / 2)) \\
& =[\ln (2 \cosh (\mathrm{H} \tau))-\mathrm{H} \tau]+2 \mathrm{H}[\tau / 2-\ln (2 \sinh (\tau / 2))]:=R_{1}(\tau)+R_{2}(\tau) .
\end{aligned}
$$

We want to find the spectrum corresponding to this limiting covariance ${ }^{4}$. Let

$$
f_{j}(u):=\frac{1}{2 \pi} \int_{-\infty}^{\infty} \mathrm{e}^{-i u \tau} R_{j}(\tau) \mathrm{d} \tau, \quad j=1,2,
$$

denote the corresponding spectral densities. In order to find the densities $f_{j}$, we use the classical relation between the differentiation and the Fourier transform,

$$
f_{j}(u)=\frac{\widehat{R_{j}^{\prime}}(u)}{\sqrt{2 \pi}(i u)}, \quad j=1,2 .
$$

\footnotetext{
${ }^{4}$ Notice however a logarithmic explosion of the term $R_{2}$ at zero. This means that the limiting process is not a usual process defined pointwise but a generalized one. This feature may not be repaired by time scaling.
} 
Since

$$
R_{1}^{\prime}(\tau)=\frac{\mathrm{H}\left(\mathrm{e}^{\mathrm{H} \tau}-\mathrm{e}^{-\mathrm{H} \tau}\right)}{\mathrm{e}^{\mathrm{H} \tau}+\mathrm{e}^{-\mathrm{H} \tau}}-\mathrm{H} \operatorname{sgn}(\mathrm{H} \tau)=\mathrm{H}(\tanh (\mathrm{H} \tau)-\operatorname{sgn}(\mathrm{H} \tau))
$$

we have

$$
\widehat{R_{1}^{\prime}}(u)=\widehat{\tanh -\operatorname{sgn}}(u / \mathrm{H}) .
$$

Furthermore, since

$$
\operatorname{tanh-\operatorname {sgn}}(u)=-i \sqrt{\pi / 2}(\sinh (\pi u / 2))^{-1}+i \sqrt{2 / \pi} u^{-1},
$$

we obtain

$$
\begin{aligned}
f_{1}(u) & =\frac{\widehat{\tanh -\operatorname{sgn}}(u / H)}{\sqrt{2 \pi}(i u)} \\
& =-(2 u \sinh (\pi u / 2 \mathrm{H}))^{-1}+\frac{\mathrm{H}}{\pi u^{2}}:=H^{-1} \phi(u / \mathrm{H}),
\end{aligned}
$$

where

$$
\phi(u)=\pi^{-1} u^{-2}\left[1-\frac{\pi u}{2 \sinh (\pi u / 2)}\right] .
$$

This is a nice function with finite limit at zero and quadratic decay at infinity.

Next, easy calculation shows that for $\tau>0$

$$
\begin{aligned}
R_{2}^{\prime}(\tau) & =\mathrm{H}(1-\operatorname{coth}(\tau / 2)) \\
& =\frac{-2 \mathrm{He}^{-\tau}}{1-\mathrm{e}^{-\tau}}=-2 \mathrm{H} \sum_{n=1}^{\infty} \mathrm{e}^{-n \tau} .
\end{aligned}
$$

Hence,

$$
R_{2}^{\prime}(\tau)=-2 \mathrm{H} \operatorname{sgn}(\tau) \sum_{n=1}^{\infty} \mathrm{e}^{-n|\tau| n}, \quad \tau \in \mathbb{R}
$$

whereas

$$
\widehat{R_{2}^{\prime}}(u)=\frac{4 \mathrm{H} i u}{\sqrt{2 \pi}} \sum_{n=1}^{\infty}\left(u^{2}+n^{2}\right)^{-1}
$$

and

$$
f_{2}(u)=\frac{\widehat{R_{2}^{\prime}}(u)}{\sqrt{2 \pi}(i u)}=\frac{2 \mathrm{H}}{\pi} \sum_{n=1}^{\infty}\left(u^{2}+n^{2}\right)^{-1} .
$$

The spectral density $f_{2}(\cdot)$ is locally nice but it decays like $|u|^{-1}$ at infinity. Therefore, it is not integrable and corresponds to a generalized Gaussian process.

By summing up, we obtain the spectral density

$$
f(u)=f_{1}(u)+f_{2}(u)=-(2 u \sinh (\pi u / 2 \mathrm{H}))^{-1}+\frac{\mathrm{H}}{\pi u^{2}}+\frac{2 \mathrm{H}}{\pi} \sum_{n=1}^{\infty}\left(u^{2}+n^{2}\right)^{-1} .
$$

Presence of hyperbolic functions in the computations suggests that there should be some relation of the introduced objects to hyperbolic geometry. This is indeed the case. Cohen and Lifshits studied in [6] many random fields and processes on the hyperbolic space. In particular, they introduced so called quadratic field playing important role in a hyperbolic version of spectral representations. As shown in ([6], Sect. 10.1), being restricted on a geodesic line of the hyperbolic plane, quadratic field generates a centered Gaussian process with stationary increments $Z(\tau), \tau \in \mathbb{R}$, with the structure function

$$
\mathbb{E} Z(\tau)^{2}=2 \ln \cosh (\tau / 2), \quad \tau \in \mathbb{R} .
$$


Notice by the way that the derivative $Z^{\prime}(\cdot)$ is a stationary process with the spectral density

$$
f_{Z}(u)=\frac{u}{2 \sinh (\pi u)} .
$$

A similar expression already appeared in (6.1).

Let us fix $H=1$ and denote $\mathbf{B}_{1}^{(1,0)}(\tau)$ a stationary Gaussian process with spectral density (6.1).

Then straightforward calculations show that independent copies of $Z(\cdot)$ and $\mathbf{B}_{1}^{(1,0)}(\cdot)$ are connected by

$$
Z(2 \tau)+\mathbf{B}_{1}^{(1,0)}(\tau)-\mathbf{B}_{1}^{(1,0)}(0)=\sqrt{2} W(\tau),
$$

where $W=W^{(1 / 2)}$ is a Wiener process.

\subsection{Case $\mathbf{H}=1$ : integral representation}

Very few is known about white noise integral representations of bfBm (for other processes, see e.g. ([11], Sect. 7.3)). We present here one for the boundary case $\mathrm{H}=1,0<\mathrm{K}<1$. By using (2.2), we have

$$
\begin{aligned}
R_{\mathrm{B}}^{(1, \mathrm{~K})}(s, t) & =2^{-\mathrm{K}}\left(\left(s^{2}+t^{2}\right)^{\mathrm{K}}-(t-s)^{2 \mathrm{~K}}\right) \\
& =\frac{\mathrm{K}}{2^{\mathrm{K}} \Gamma(1-\mathrm{K})} \int_{0}^{\infty}\left(\mathrm{e}^{-x(t-s)^{2}}-\mathrm{e}^{-x\left(s^{2}+t^{2}\right)}\right) x^{-1-\mathrm{K}} \mathrm{d} x \\
& =\frac{\mathrm{K}}{2^{\mathrm{K}} \Gamma(1-\mathrm{K})} \int_{0}^{\infty}\left(\mathrm{e}^{-x\left(s^{2}+t^{2}\right)}\left(\mathrm{e}^{2 x s t}-1\right)\right) x^{-1-\mathrm{K}} \mathrm{d} x \\
& =\frac{\mathrm{K}}{2^{\mathrm{K}} \Gamma(1-\mathrm{K})} \sum_{n=1}^{\infty} \int_{0}^{\infty}\left(\mathrm{e}^{-x\left(s^{2}+t^{2}\right)} \frac{(2 x s t)^{n}}{n !}\right) x^{-1-\mathrm{K}} \mathrm{d} x .
\end{aligned}
$$

It follows that

$$
\mathbf{B}^{(1, \mathrm{~K})}(s)=\left(\frac{\mathrm{K}}{2^{\mathrm{K}} \Gamma(1-\mathrm{K})}\right)^{1 / 2} \sum_{n=1}^{\infty} s^{n}\left(\frac{2^{n}}{n !}\right)^{1 / 2} \int_{0}^{\infty} \mathrm{e}^{-x s^{2}} x^{(n-1-\mathrm{K}) / 2} \mathcal{W}_{n}(\mathrm{~d} x),
$$

where $\mathcal{W}_{n}$ are independent Gaussian white noises on $\mathbb{R}_{+}$controlled by Lebesgue measure.

\section{A POSTERIOR DISCUSSION}

One of the referees made some remarks related to a more general context than the particular results of this note. We also believe that a wider discussion might be interesting to the reader.

The referee states "Since the introduction of bfBm there is a real problem of motivation. Apart from selfsimilarity, this process enjoys no inherent property, and there are lots of processes with the same qualitative behaviors: a lot of Bernstein functions and functions $\sigma$ can be used and, with the help of of Proposition 2.1, new processes may be introduced". We basically agree with that, although we find that self-similarity is quite a strong additional feature for picking bfBm from the crowd of possible generalizations of fBm. In our opinion, the decisive argument for motivation of the interest would be finding some natural models converging to the studied process. So far, only the case $\mathrm{H}=\frac{1}{2}$ of bfBm appeared in this setting but, as we noticed (may be for the first time?), this case is directly related to $\mathrm{fBm}$ itself and, therefore, may not advocate for the entire family of bfBm's.

Back to bfBm, the referee remarks correctly that our existence results are not the same for $\mathbb{R}$ and $\mathbb{R}_{+}$. This is of course the weakness of our spectral approach (that is focused only on $\mathbb{R}_{+}$) and should be considered as a source of open problems. Moreover, considering multivariate case only makes sense when the existence of bfBm on the whole $\mathbb{R}$ will be understood. Solving these problems apparently goes far beyond the means of our note. 
Acknowledgements. We are very grateful to both anonymous referees for the careful reading of our note and for their insightful remarks.

\section{REFERENCES}

[1] M. Abramowitz and I. Stegun, Handbook of Mathematical Functions. Dover Publ., New York (1972).

[2] X. Bardina and K. Es-Sebaiy, An extension of bifractional Brownian motion. Commun. Stoch. Anal. 5 (2011) 333-340.

[3] O.E. Barndorff-Nielsen and V. Perez-Abreu, Stationary and selfsimilar processes driven by Lévy processes. Stochastic Processes Appl. 84 (1999) 357-369.

[4] T. Bojdecki, L.G. Gorostiza and A. Talarczyk, Some extensions of fractional Brownian motion and sub-fractional Brownian motion related to particle systems. Electron. Commun. Probab. 12 (2007) 161-172.

[5] P. Cheredito, W. Kawaguchi and M. Maejima, Fractional Ornstein-Uhlenbeck processes. Electron. J. Probab. 8 (2003) 1-14.

[6] M. Lifshits and S. Cohen, Stationary Gaussian random fields on hyperbolic spaces and on Euclidean spheres. ESAIM: PS 16 (2012) 165-221.

[7] D.S. Egorov, Annual student's memoir. St. Petersburg State University (2014).

[8] P. Embrechts and M. Maejima, Selfsimilar Processes. Princeton University Press (2002).

[9] C. Houdré and J. Villa, An example of infinite dimensional quasi-helix. Stochastic Models. Ser.: Contemp. Math. 336 (2003) 195-201.

[10] P. Lei and D. Nualart, A decomposition of the bifractional Brownian motion and some applications. Stat. Probab. Lett. 79 (2009) 619-624.

[11] M. Lifshits, Random Processes by Example. World Scientific, Singapore (2014).

[12] M. Lifshits and K. Volkova, Bifractional Brownian motion: existence and border cases. Preprint arXiv:1502.02217 (2015).

[13] M.A. Lifshits, R. Schilling and I. Tyurin, A probabilistic inequality related to negative definite functions, in High Dimensional Probability VI, Vol. 66 of Ser. Progress in Probability, edited by C. Houdré et al. Birkhäuser, Basel. Preprint: arXiv: 1205.1284 (2013).

[14] C. Ma, The Schoenberg-Lévy kernel and relationships among fractional Brownian motion, bifractional Brownian motion and others. Theory Probab. Appl. 57 (2013) 619-632.

[15] M. Marouby, Micropulses and different types of Brownian motion. J. Appl. Probab. 48, (2011) 792-810.

[16] R. Schilling, R. Song and Z. Vondraček, Bernstein Functions. De Gruyter, Berlin. Stud. Math. (2010) 37.

[17] G. Samorodnitsky and M.S. Taqqu, Stable Non-Gaussian Random Processes. Chapman \& Hall, New York (1994).

[18] C.A. Tudor and Y. Xiao, Sample path properties of bifractional Brownian motion. Bernoulli 13 (2007) 1023-1052.

[19] W. Wang, On p-variation of bifractional Brownian motion. Appl. Math. J. Chinese Univ. 26 (2011) $127-141$. 